\title{
Qualidade de solo submetido a sistemas de cultivo com preparo convencional e plantio direto
}

\author{
Eusângela Antônia Costa(1), Wenceslau J. Goedert( ${ }^{(2)}$ e Djalma Martinhão Gomes de Sousa( ${ }^{(3)}$
}

\begin{abstract}
(1)Empresa de Assistência Técnica e Extensão Rural, Caixa Postal 10.748, CEP 73330-023 Planaltina, DF. E-mail: eusangela@hotmail.com (2)Universidade de Brasília, Caixa Postal 04508, CEP 70710-900 Brasília, DF. E-mail: goedert@unb.br (3)Embrapa Cerrados, Caixa Postal 08223, CEP 73310-970 Planaltina, DF. E-mail: dmgsousa@cpac.embrapa.br
\end{abstract}

Resumo - O objetivo deste trabalho foi avaliar a qualidade de um Latossolo Vermelho submetido a sistemas de cultivo com preparo convencional e plantio direto. Foram estudadas duas áreas experimentais, localizadas na Embrapa Cerrados, em Planaltina, DF, com oito e dez anos de cultivo. Foram coletadas amostras de solo, em diversas profundidades, nas parcelas experimentais e em área de cerrado nativo. Os seguintes atributos foram avaliados: densidade do solo, porosidade total, capacidade de água disponível, grau de floculação, resistência do solo à penetração, teor de matéria orgânica, capacidade de troca catiônica, fósforo remanescente, carbono da biomassa microbiana e respiração basal. Os dados obtidos foram comparados a valores referenciais quanto à qualidade do solo, mediante modelagem gráfica. Observou-se que a qualidade do solo, em ambos os sistemas de cultivo, é similar quanto aos atributos físicos; os teores de matéria orgânica e fósforo remanescente também são semelhantes, mas a capacidade de troca catiônica é mais alta no solo sob plantio direto. Em relação aos atributos biológicos, o solo sob plantio direto apresenta atividade biológica mais elevada. A qualidade do solo em ambos os sistemas é similar, em relação aos atributos avaliados.

Termos para indexação: física do solo, química do solo, biologia do solo, Latossolo, Cerrado.

\section{Soil quality under tillage and no-tillage cropping systems}

\begin{abstract}
The objective of this study was to evaluate the quality of an Oxisol under tillage and no-tillage systems. Two experimental areas were studied, both located in Embrapa Cerrados, Planaltina, DF, Brazil, with eight and ten years of cropping. Soil samples were collected from different depth layers in the experimental plots and native cerrado vegetation area. The following soil atributes were evaluated: bulk density, soil porosity, available water capacity, degree of flocculation, soil resistance to penetration, organic matter content, cation exchange capacity, equilibrium phosphorus, microbial biomass carbon and basal respiration. The data obtained were compared with referential values related to soil quality. The soil quality under both systems is similar, considering the soil physical attributes. The values for organic matter and equilibrium phosphorus are also similar, but the cation exchange capacity is higher for soil under no-tillage. Concerning the biological attributes, the soil under no-tillage presents higher biological activity. The soil quality is similar, under both cropping systems.
\end{abstract}

Index terms: soil physics, soil chemistry, soil biology, Oxissol, Cerrado.

\section{Introdução}

Há 30 anos teve início a incorporação de áreas da Região do Cerrado para a produção de grãos. Esse processo ocorreu com o emprego de sistemas de preparo intensivo do solo, tendo-se em vista a necessidade de incorporação de restos da vegetação nativa, de corretivos e de fertilizantes. Embora se verifique grande variação de processos de preparo do solo, tais processos têm sido, genericamente, denominados como sistemas de preparo convencional.
O revolvimento contínuo e intenso do solo pode resultar em diminuição de sua qualidade. Segundo Seguy et al. (1984), os principais efeitos são causados, potencialmente, pela pulverização do solo, que aumenta a exposição dos compostos orgânicos, e pelo favorecimento de condições para a formação de uma zona compactada abaixo da camada arada.

Tais observações resultaram em preocupação com a qualidade do solo, entendida, resumidamente, como sua capacidade de manter uma produção de modo sustentável. 
Uma alternativa para minimizar a mobilização do solo foi a introdução, na região, há cerca de 15 anos, do sistema de plantio direto, que preconiza a semeadura em solo coberto por palha, com o mínimo de mobilização na linha de semeadura, para que o solo permaneça mais protegido do impacto das gotas de chuva.

Como conseqüência, tem crescido o interesse em se avaliar a qualidade do solo submetido a diferentes processos de cultivo, a fim de definir a tecnologia mais racional de uso do solo. Vários trabalhos compararam a qualidade de solos tropicais sob diferentes sistemas agrícolas, podendo-se destacar: Maria et al. (1999), Silva et al. (2000), Beutler et al. (2001), Stone \& Silveira (2001), D’Andréa et al. (2002), Goedert et al. (2002), Mendes et al. (2003), Oliveira et al. (2004) e Dufranc et al. (2004). Tem-se observado que a qualidade do solo é superior em sistemas em que a sua mobilização é pequena.

Quantificar a qualidade do solo não é tarefa fácil; a dificuldade advém do fato de que a qualidade do solo depende de suas propriedades intrínsecas, de suas interações com o ecossistema e, ainda, de prioridades de uso, influenciadas inclusive, por aspectos socioeconômicos e políticos.

O objetivo deste trabalho foi avaliar a qualidade de um Latossolo Vermelho, submetido a sistemas de cultivo com preparo convencional (SPC) e plantio direto (SPD), por oito e dez anos, mediante a adoção de modelo de representação gráfica.

\section{Material e Métodos}

A pesquisa foi realizada tendo-se como base dois experimentos instalados na Embrapa Cerrados, em Planaltina, DF, a 15 36'S, 470 42'W e altitude de $1.014 \mathrm{~m}$. O clima é do tipo Cwa, com precipitação pluvial média anual de $1.570 \mathrm{~mm}$ e temperatura média anual de $21,3^{\circ} \mathrm{C}$. A topografia é plana e originalmente coberta por vegetação de cerrado, e predomina um solo profundo, argiloso e bem drenado, classificado como Latossolo Vermelho distrófico (LV).

Os experimentos foram instalados em 1994 e 1996, tendo como objetivo a avaliação agronômica de fontes e níveis de $\mathrm{P}$, sob dois sistemas de cultivo: preparo convencional e plantio direto. $\mathrm{Na}$ implantação desses experimentos, as áreas foram corrigidas com calcário, para obtenção de saturação por bases de $50 \%$.
A adubação com os demais nutrientes foi a recomendada para o desenvolvimento de soja ou milho, de acordo com Sousa \& Lobato (2004).

Para a realização dessa pesquisa, foram selecionadas quatro parcelas experimentais, com três repetições, optando-se pelas parcelas que receberam adubação fosfatada na forma de superfosfato triplo, aplicado no sulco de plantio. Adicionalmente, foi avaliado o solo sob vegetação natural de cerrado (CE), em área contígua aos experimentos.

Duas parcelas fazem parte do experimento instalado há dez anos, em área de solo sob cerrado, uma com preparo convencional (SPC 10) e outra com plantio direto (SPD 10). O preparo convencional do solo consistiu numa aração e uma gradagem niveladora. O delineamento experimental foi de blocos ao acaso. Foi cultivada soja durante nove anos, e milho no décimo, sendo que antes do milho foi semeado milheto como planta de cobertura. A produtividade média da soja (nove cultivos) no SPD foi de 2,7 t ha-1 e no SPC foi de 2,6 $\mathrm{t} \mathrm{ha}^{-1}$, enquanto que a do milho (2003/2004) foi de 9,9 e 9,2 $\mathrm{t} \mathrm{ha}^{-1}$, respectivamente. A produção de restos culturais, inclusive cultivos e milheto, foi de 48,3 t ha ${ }^{-1}$ de matéria seca no SPD e 46,2 $\mathrm{t} \mathrm{ha}^{-1}$ no SPC.

As outras duas parcelas fazem parte de experimento instalado há oito anos, em área de solo já em cultivo por cerca de 20 anos, sendo uma com preparo convencional (SPC 8) e outra com plantio direto (SPD 8). O delineamento experimental foi um fatorial com blocos ao acaso. O esquema de cultivo envolveu uma rotação anual de milho e soja, tendo-se, anualmente, o milheto como planta de cobertura. A produtividade média da soja (quatro cultivos) foi de $3,5 \mathrm{t} \mathrm{ha}^{-1}$, e a do milho (quatro cultivos) foi de $11,2 \mathrm{t} \mathrm{ha}^{-1}$, não tendo havido diferença entre os sistemas de cultivo. A produção de restos culturais, inclusive cultivos e milheto, foi de 107,2 t ha-1 de matéria seca no SPD e 104,0 t ha ${ }^{-1}$ no SPC.

As amostras compostas de solo foram coletadas com trado, em duas camadas de solo, $0-10 \mathrm{~cm}$ e $10-20 \mathrm{~cm}$, para realização das análises físicas e químicas; e na camada de 0-10 cm para realização das análises biológicas, com três repetições e 15 subamostras, em abril de 2004, final da estação chuvosa.

Adicionalmente, amostras indeformadas foram coletadas para determinação da densidade, porosidade e capacidade de água disponível, em quatro camadas de solo, 0-5, 5-10, 10-20 e 20-30 cm, com três repetições por parcela. 
Medidas diretas de resistência do solo à penetração foram executadas em todas as parcelas, num total de 15 pontos por parcela, em novembro de 2004. A umidade gravimétrica do solo, por ocasião das medidas, variou de 28 a $31 \%$.

Para avaliar a qualidade do solo das parcelas experimentais e do CE, foram quantificados os seguintes atributos: densidade do solo, porosidade total, grau de floculação, resistência do solo à penetração, capacidade de água disponível, teor de matéria orgânica, capacidade de troca catiônica, fósforo remanescente, carbono total da biomassa microbiana e respiração basal. Para quantificar os atributos, foram adotados os procedimentos tradicionalmente utilizados na pesquisa (Tabela 1).

As análises estatísticas dos dados mensurados foram realizadas com o software estatístico SAS, e a análise de variância e a comparação entre as médias foram feitas pelo teste de Tukey a 5\% de probabilidade.

Para comparar a qualidade do solo, de forma integrada, sob os dois sistemas de cultivo, foram organizados modelos gráficos, do tipo radial, em que cada atributo é locado em um dos seus raios. Para tanto, foram definidos, inicialmente, valores referenciais com índice 1, para cada atributo (Tabela 1). Esses valores representam níveis considerados sustentáveis para aquele atributo. A seguir, calculou-se o quociente entre o valor obtido pela análise e o valor referencial. Nos casos em que o valor sustentável deveria ser menor do que o referencial (densidade do solo e resistência à penetração), utilizouse o inverso do valor determinado pela análise.

Para a construção dos gráficos radiais, foram considerados os valores obtidos na profundidade de $0-10 \mathrm{~cm}$, com exceção do atributo resistência do solo à penetração, para o qual se optou pelos valores obtidos na camada de 20-30 cm, por ser essa a camada considerada mais crucial no que concerne aos efeitos da compactação do solo.

\section{Resultados e Discussão}

Os valores médios de densidade do solo (Ds), obtidos nas parcelas experimentais submetidas aos dois sistemas de cultivo, oscilaram entre 0,92 e 1,08 $\mathrm{g} \mathrm{cm}^{-3}$, considerando-se todas as camadas avaliadas (Tabela 2). Em termos gerais, esses valores podem ser considerados normais para latossolos argilosos sob cultivo (Maria et al., 1999; Araújo, 2004).

Não houve diferença significativa entre os sistemas de cultivo, o que indica que tais sistemas de uso afetaram a Ds de modo similar. Observa-se uma elevação deste atributo no SPC e SPD, quando comparado com o solo sob CE, fato esperado tendo-se em vista o revolvimento do solo e o tráfego de implementos sobre a superfície do terreno.

Analisando-se a Ds em profundidade (Tabela 2), observam-se valores mais altos nas camadas de 10-20 e 20-30 cm, em função, provavelmente, do menor conteúdo de matéria orgânica e do acúmulo das pressões exercidas pelo tráfego nestas camadas. Contudo, os dados se situam em torno de $1 \mathrm{~g} \mathrm{~cm}^{-3}$, valor considerado como referência (Tabela 1).

Não houve compactação do solo, e pode-se inferir que os sistemas de manejo avaliados afetaram de modo similar a Ds. Tais resultados diferem de alguns traba-

Tabela 1. Atributos avaliados, profundidades amostradas, métodos utilizados e valores de referência adotados.

\begin{tabular}{|c|c|c|c|}
\hline Atributo & Camada $(\mathrm{cm})$ & Método & Valor de referência $^{(1)}$ \\
\hline Densidade do solo & $0-5,5-10,10-20$ e $20-30$ & Anel volumétrico (Embrapa, 1997) & $1 \mathrm{~g} \mathrm{~cm}^{-3}$ \\
\hline Porosidade total & $0-5,5-10,10-20$ e $20-30$ & Método da centrífuga (Freitas Júnior \& Silva, 1984) & $0,60 \mathrm{~cm}^{3} \mathrm{~cm}^{-3}$ \\
\hline Capacidade de água disponível & $0-5,5-10,10-20$ e $20-30$ & Método da centrífuga (Freitas Júnior \& Silva, 1984) & $13 \mathrm{~cm}^{3} \mathrm{~cm}^{-3}$ \\
\hline Resistência do solo à penetração & De 5 em $5 \mathrm{~cm}$, até $45 \mathrm{~cm}$ & $\begin{array}{l}\text { Penetrômetro de pressão, com ponteira de } 1 \mathrm{~cm} \text { de } \\
\text { diâmetro }\end{array}$ & $1 \mathrm{MPa}$ \\
\hline Grau de floculação & $0-10$ e $10-20$ & Argila dispersa em água (Embrapa, 1997) & $50 \%$ \\
\hline Matéria orgânica & $0-10$ e $10-20$ & Método de oxidação via úmida (Embrapa, 1997) & 4,0 dag $\mathrm{kg}^{-3}$ \\
\hline Capacidade de troca catiônica & $0-10$ e $10-20$ & $\begin{array}{l}\text { Soma de cátions trocáveis com }\left(\mathrm{H}+\mathrm{Al}^{3+}\right) \text { (Embrapa, } \\
\text { 1997) }\end{array}$ & $10 \mathrm{cmol}_{\mathrm{c}} \mathrm{dm}^{-3}$ \\
\hline P remanescente & $0-10$ e $10-20$ & Alvarez Venegas et al. (1999) & $30 \mathrm{mg} \mathrm{kg}^{-1}$ \\
\hline $\begin{array}{l}\text { Carbono da biomassa } \\
\text { microbiana }\end{array}$ & $0-10$ & $\begin{array}{l}\text { Clorofórmio-fumigação-incubação (Jenkinson \& } \\
\text { Powlson, 1976) }\end{array}$ & $400 \mathrm{mg} \mathrm{C} \mathrm{kg}^{-1}$ \\
\hline Respiração basal & $0-10$ & $\begin{array}{l}\text { Clorofórmio-fumigação-incubação (Jenkinson \& } \\
\text { Powlson, 1976) }\end{array}$ & $80 \mathrm{mg} \mathrm{C} \mathrm{kg}^{-1}$ \\
\hline
\end{tabular}

(1)Para a camada de $0-10 \mathrm{~cm}$. 
lhos, em que foi registrado um adensamento superficial no SPD, e subsuperficial no SPC (Maria et al., 1999; Stone \& Silveira, 2001). Uma possível justificativa para os resultados obtidos neste trabalho é o manejo utilizado nas parcelas experimentais, principalmente o pouco revolvimento do solo no SPC (uma aração e uma gradagem niveladora, por ano) e a manutenção de plantas de cobertura (milheto) em todas as parcelas.

A porosidade total do solo $(\mathrm{Pt})$, propriedade que tem uma relação estreita e inversa com a Ds, apresentou valores médios muito próximos nos dois sistemas de uso do solo, o que demonstra que o cultivo afetou de modo similar esse atributo (Tabela 2).

A Pt diminuiu com a profundidade, e foi estatisticamente menor a partir da profundidade de $10 \mathrm{~cm}$, possivelmente em função do maior teor de matéria orgânica na camada superficial e do acúmulo das pressões abaixo de $10 \mathrm{~cm}$ de profundidade, resultante do tráfego.

No que se refere à capacidade de água disponível (CAD), os valores obtidos variaram entre 10,5 e $13,9 \mathrm{~cm} \mathrm{~cm}^{-3}$, e não foi observada diferença estatística entre os sistemas e as profundidades. Oliveira et al. (2004) avaliaram um solo, depois de 20 anos sob cultivo, e concluíram que o sistema de semeadura direta apresentou maior disponibilidade de água na camada de $0-5 \mathrm{~cm}$. Possivelmente a variação deste atributo seja dependente do tempo de cultivo.

Os resultados obtidos para resistência do solo à penetração (Rp) (Tabela 3) variaram de 0,09 a 1,96 MPa e podem ser considerados dentro de uma amplitude normal para solos cultivados. Valores com magnitude similar foram relatados por Araújo (2004) e Secco et al. (2004), para latossolos submetidos ao preparo convencional.

Observa-se, ainda, que a Rp, nas parcelas sob SPC, é mais baixa na camada superficial $(0-20 \mathrm{~cm})$ e mais alta a partir dessa profundidade, enquanto nas parcelas sob SPD ocorre o inverso (Tabela 3). O aumento dos valores de Rp, na camada de $20-25 \mathrm{~cm}$, para o SPC, pode indicar início de formação de pé-de-grade, provocada pelo preparo contínuo da camada superficial. Efeitos similares foram relatados por Maria et al. (1999) e Goedert et al. (2002).

Tabela 2. Valores médios de densidade do solo e porosidade total, em quatro camadas de solo, em áreas cultivadas sob sistema de preparo convencional (SPC) e plantio direto (SPD), por períodos de dez e oito anos, e em área de Cerrado (CE) ${ }^{(1)}$.

\begin{tabular}{llcc}
\hline Sistema de cultivo & \multicolumn{2}{c}{ Camadas de solo $(\mathrm{cm})$} \\
\cline { 2 - 4 } & $0-5$ & $5-10$ & $10-20$ \\
SPC 10 & $0,98 \mathrm{aB}$ & Densidade do solo $\left(\mathrm{g} \mathrm{cm}^{-3}\right)$ & $1,02 \mathrm{bAB}$ \\
SPD 10 & $0,92 \mathrm{abB}$ & $0,99 \mathrm{bcB}$ & $1,02 \mathrm{bA}$ \\
SPC 8 & $0,98 \mathrm{aB}$ & $1,00 \mathrm{bcA}$ & $1,06 \mathrm{aA}$ \\
SPD 8 & $0,98 \mathrm{aB}$ & $0,98 \mathrm{cB}$ & $1,06 \mathrm{aA}$ \\
CE & $0,82 \mathrm{bB}$ & $1,02 \mathrm{abcAB}$ & $0,91 \mathrm{cA}$ \\
SPC 10 & & $0,87 \mathrm{dAB}$ & $1,06 \mathrm{aA}$ \\
SPD 10 & $0,55 \mathrm{bcA}$ & Porosidade total $\left(\mathrm{cm}^{3} \mathrm{~cm}^{-3}\right)$ & $0,06 \mathrm{aA}$ \\
SPC 8 & $0,59 \mathrm{bA}$ & $0,55 \mathrm{bA}$ & $0,52 \mathrm{bB}$ \\
SPD 8 & $0,55 \mathrm{bcA}$ & $0,54 \mathrm{bAB}$ & $0,52 \mathrm{bB}$ \\
CE & $0,56 \mathrm{bcA}$ & $0,54 \mathrm{bA}$ & $0,49 \mathrm{bB}$ \\
\hline
\end{tabular}

(1)Médias seguidas de letras iguais, minúsculas na coluna e maiúsculas na linha, não diferem entre si pelo teste de Tukey a 5\% de probabilidade.

Tabela 3. Valores médios de resistência do solo à penetração (MPa), em camadas de solo, em áreas cultivadas sob sistema de preparo convencional (SPC) e plantio direto (SPD), por períodos de dez e oito anos, e em área de Cerrado (CE) (1).

\begin{tabular}{|c|c|c|c|c|c|c|c|c|c|}
\hline \multirow{2}{*}{$\begin{array}{l}\text { Sistema de } \\
\text { cultivo }\end{array}$} & \multicolumn{9}{|c|}{ Camada de solo $(\mathrm{cm})$} \\
\hline & $0-5$ & $5-10$ & $10-15$ & $15-20$ & $20-25$ & $25-30$ & $30-35$ & $35-40$ & $40-45$ \\
\hline $\mathrm{SPC} 10$ & $0,15 \mathrm{cdD}$ & $0,24 \mathrm{dD}$ & $0,35 \mathrm{cD}$ & $0,92 \mathrm{bcC}$ & $1,65 \mathrm{bcAB}$ & $1,96 \mathrm{aA}$ & $1,75 \mathrm{aAB}$ & $1,39 \mathrm{aBC}$ & $1,19 \mathrm{aBC}$ \\
\hline SPD 10 & $1,03 \mathrm{aC}$ & $1,25 \mathrm{abBC}$ & $1,67 \mathrm{aAB}$ & $1,91 \mathrm{aA}$ & $1,81 \mathrm{aAB}$ & $1,65 \mathrm{aAB}$ & $1,27 \mathrm{bcBC}$ & $1,15 \mathrm{aC}$ & $0,99 \mathrm{abC}$ \\
\hline SPC 8 & $0,09 \mathrm{dC}$ & $0,25 \mathrm{dC}$ & $0,36 \mathrm{cD}$ & $1,03 \mathrm{bB}$ & $1,73 \mathrm{abA}$ & $1,73 \mathrm{aA}$ & $1,57 \mathrm{abAB}$ & $1,23 \mathrm{aB}$ & $1,00 \mathrm{abB}$ \\
\hline SPD 8 & $0,64 \mathrm{bcD}$ & $1,40 \mathrm{aBC}$ & $1,73 \mathrm{aAB}$ & $1,69 \mathrm{aAB}$ & $1,91 \mathrm{aA}$ & $1,65 \mathrm{aAB}$ & $1,52 \mathrm{abBC}$ & $1,29 \mathrm{aBC}$ & $1,15 \mathrm{aC}$ \\
\hline $\mathrm{CE}$ & $0,73 \mathrm{bC}$ & $1,02 \mathrm{cAB}$ & $1,07 \mathrm{bA}$ & $1,09 \mathrm{bA}$ & $1,03 \mathrm{cA}$ & $0,93 \mathrm{bAB}$ & $0,86 \mathrm{cBC}$ & $0,76 \mathrm{bBC}$ & $0,68 \mathrm{cC}$ \\
\hline
\end{tabular}

(1)Médias seguidas de letras iguais, minúsculas na coluna e maiúsculas na linha, não diferem entre si pelo teste de Tukey a 5\% de probabilidade. 
Altos valores de Rp podem restringir a percolação da água e prejudicar o crescimento das raízes, com reflexos na produtividade das culturas. Tendo-se em vista a variabilidade intrínseca deste atributo, não existe consenso sobre o valor acima do qual ocorrem restrições. Contudo, valores de Rp maiores que 2 MPa têm sido considerados fortemente restritivos ao crescimento das raízes das plantas (Beutler et al., 2003).

Os valores de grau de floculação (Gf) variaram entre 38 e 44\%, e não diferiram estatisticamente entre si, o que indica que tal atributo é pouco sensível às variações de manejo.

Os teores médios de matéria orgânica (MO) variaram de 2,71 a 3,30 dag kg-1, na camada $0-10 \mathrm{~cm}$, e de 2,20 a 2,57 dag kg-1 na camada $10-20 \mathrm{~cm}$, nas parcelas sob SPC e SPD (Tabela 4).

Houve diferença estatística entre os teores de MO nas camadas de $0-10$ e 10-20 cm nas parcelas sob SPD, enquanto sob SPC não houve diferença entre as profundidades. Tal acúmulo de MO, mais pronunciado na camada de $0-10 \mathrm{~cm}$ da parcela com oito anos de cultivo, se deve à manutenção dos restos vegetais na superfície do terreno no SPD e sua incorporação no SPC.

Conforme Tabela 4, a capacidade de troca catiônica (CTC) variou de 9,6 a $11,9 \mathrm{cmol}_{\mathrm{C}} \mathrm{dm}^{-3}$ na camada 0-10 cm, e de 9,0 a $10,2 \mathrm{cmol}_{\mathrm{c}} \mathrm{dm}^{-3}$ na $10-20 \mathrm{~cm}$, situando-se próxima ao valor considerado como sustentável para latossolos de textura argilosa (Tabela 1).

Como a maior proporção da CTC de solos com argilas de baixa atividade é originária de sua fração orgânica, era esperado que as tendências registradas para a MO se mantivessem para a CTC. Observa-se um valor de CTC maior na camada de $0-10 \mathrm{~cm}$, nas parcelas sob SPD, enquanto sob SPC não foi detectada diferença em profundidade.

$\mathrm{O}$ teor de $\mathrm{P}$ remanescente (P-rem) foi incluído no conjunto de atributos, a fim de se avaliar a capacidade do solo em adsorver íons fosfato em formas não lábeis, indicador importante para orientar a adubação fosfatada. Os resultados obtidos para esse atributo mostram pouca variação entre os sistemas de manejo avaliados, os valores se situaram em torno de $30 \mathrm{mg} \mathrm{kg}^{-1}$ (Tabela 4).

Com relação ao carbono da biomassa microbiana (CBM) verificou-se variação de 228 a $358 \mathrm{mg} \mathrm{kg}^{-1}$, nas parcelas sob SPC e SPD, tendo sido registrado coeficiente de variação de 17\% (Tabela 5). Os valores encontrados estão dentro de um intervalo relatado na literatura, para latossolos sob cultivo em SPD e SPC (Dufranc et al., 2004). Balota et al. (1998) e Mendes et al. (2003) verificaram tendência de valores maiores de CBM em solo sob SPD, o que indica que esse sistema favorece a atividade biológica do solo.

A respiração basal (Rb), que reflete a taxa de liberação de $\mathrm{CO}_{2}$ durante 17 dias, também apresentou alta variação nas parcelas sob SPC e SPD, com valores entre 35 e $116 \mathrm{mg} \mathrm{C} \mathrm{kg}^{-1}$, e o valor mais elevado foi registrado no tratamento SPD 8 (Tabela 5), possivelmente em resposta à maior adição de restos culturais com maior relação C/N (milho).

Foi observada atividade biológica mais alta no solo cultivado sob SPD, possivelmente como resultante do maior teor de MO presente. Observações similares foram relatadas por D’Andréa et al. (2002), para áreas de cerrado sob cultivo, no Estado de Goiás.

Na comparação entre os solos cultivados por um período de dez anos sob SPC e SPD, reunindo-se todos os atributos avaliados em gráficos radiais (Figura 1), observa-se que a qualidade destes solos é muito similar, principalmente em relação aos atributos físicos.

Os teores de MO e de P-rem também são similares, mas a CTC é mais alta no solo sob SPD, o que indica que a manutenção dos restos vegetais sobre a superfície do terreno está contribuindo diferencialmente para o aumento da CTC nesse sistema.

Tabela 4. Valores médios dos atributos químicos, avaliados em duas camadas de solo, em áreas cultivadas sob sistema de preparo convencional (SPC) e plantio direto (SPD), por períodos de dez e oito anos, e em área de Cerrado (CE) ${ }^{(1)}$.

\begin{tabular}{|c|c|c|c|c|c|c|}
\hline \multirow[t]{2}{*}{ Sistema de cultivo } & \multicolumn{2}{|c|}{ Matéria orgânica (dag kg $\left.{ }^{-1}\right)$} & \multicolumn{2}{|c|}{ CTC $\left(\mathrm{cmol}_{\mathrm{c}} \mathrm{dm}^{-3}\right)$} & \multicolumn{2}{|c|}{ P-rem $\left(\mathrm{mg} \mathrm{kg}^{-1}\right)$} \\
\hline & $0-10 \mathrm{~cm}$ & $10-20 \mathrm{~cm}$ & $0-10 \mathrm{~cm}$ & $10-20 \mathrm{~cm}$ & $0-10 \mathrm{~cm}$ & $10-20 \mathrm{~cm}$ \\
\hline SPC 10 & $2,71 \mathrm{cA}$ & $2,57 \mathrm{abA}$ & $9,6 \mathrm{cA}$ & $9,1 \mathrm{abA}$ & $33,6 \mathrm{abA}$ & $35,2 \mathrm{aA}$ \\
\hline SPD 10 & $2,84 \mathrm{bcA}$ & $2,20 \mathrm{bcB}$ & $10,9 \mathrm{bcA}$ & $9,0 \mathrm{abB}$ & $32,5 \mathrm{abA}$ & $31,7 \mathrm{aA}$ \\
\hline SPC 8 & $2,71 \mathrm{cA}$ & $2,56 \mathrm{abA}$ & $10,4 \mathrm{bcA}$ & $10,2 \mathrm{aA}$ & $33,7 \mathrm{abA}$ & $31,6 \mathrm{aA}$ \\
\hline SPD 8 & 3,30abA & $2,39 \mathrm{bcB}$ & $11,9 \mathrm{aA}$ & 9,9abB & $36,0 \mathrm{aA}$ & $30,6 \mathrm{aA}$ \\
\hline $\mathrm{CE}$ & $3,36 \mathrm{aA}$ & $2,75 \mathrm{aB}$ & $11,2 \mathrm{aA}$ & $10,0 \mathrm{aA}$ & $25,7 \mathrm{bA}$ & $15,9 \mathrm{bB}$ \\
\hline
\end{tabular}

${ }^{(1)}$ Médias seguidas de letras iguais, minúsculas na coluna e maiúsculas na linha, não diferem entre si pelo teste de Tukey a 5\% de probabilidade. 
Em relação aos atributos biológicos, o solo sob SPD apresenta valor médio superior de CBM, embora não apresente a mesma tendência para a respiração basal, o que seria esperado.

Para a área sob cultivo há oito anos, observa-se também a semelhança entre os polígonos para os dois sistemas de cultivo, com índices coincidentes para os atributos físicos, e com tendências similares à área sob cultivo há dez anos, no que se refere aos demais atributos (Figura 1). Contudo, cabe destacar a elevada atividade biológica detectada no solo sob SPD, que confirma dados relatados por Balota et al. (1998) e Mendes et al. (2003).

Numa avaliação geral e conjunta dos gráficos radiais, verifica-se que a qualidade do solo, sob os dois sistemas de cultivo, é muito semelhante, podendo-se destacar apenas a mais alta atividade biológica no solo sob SPD (Figura 1). Adicionalmente, observa-se que os polígonos formados são de magnitude e formato similares ao formado pelos valores de referência. $\mathrm{O}$ fato de que a produtividade das culturas tem sido elevada e de magnitude similar, nos dois sistemas de cultivo, já era um forte indicativo dessa conclusão.

A visualização da qualidade do solo mediante modelos gráficos, considerando-se um conjunto de indicadores, constitui-se num instrumento útil para orientar o manejo racional de uma área sob cultivo.

Embora o objetivo desta pesquisa seja a avaliação comparativa da qualidade de solo, submetido a dois sistemas de cultivo, os atributos foram também medidos em solo sob CE. Uma análise dos dados obtidos evidencia que o uso do solo modifica, significativamente, suas propriedades originais (Tabelas 2 a 5). Tal efeito é esperado, tendo-se em vista que o equilíbrio do solo sob

Tabela 5. Valores médios de carbono da biomassa microbiana (CBM) e respiração basal (Rb), avaliados na camada de solo de 0-10 cm, em áreas cultivadas sob sistema de preparo convencional (SPC) e plantio direto (SPD), por períodos de dez e oito anos, e em área de Cerrado (CE). O valor da Rb se refere a um período de 17 dias de incubação(1).

\begin{tabular}{lcc}
\hline Sistema de cultivo & $\mathrm{CBM}\left(\mathrm{mg} \mathrm{C} \mathrm{kg}^{-1}\right)$ & $\mathrm{Rb}\left(\mathrm{mg} \mathrm{C} \mathrm{kg}^{-1}\right)$ \\
\hline SPC 10 & $228 \mathrm{~d}$ & $54 \mathrm{bc}$ \\
SPD 10 & $358 \mathrm{bcd}$ & $58 \mathrm{bc}$ \\
SPC 8 & $310 \mathrm{bc}$ & $35 \mathrm{c}$ \\
SPD 8 & $256 \mathrm{~cd}$ & $116 \mathrm{a}$ \\
CE & $487 \mathrm{a}$ & $82 \mathrm{ab}$ \\
\hline
\end{tabular}

(1)Médias seguidas de letras iguais, na coluna, não diferem entre si pelo teste de Tukey a $5 \%$ de probabilidade.
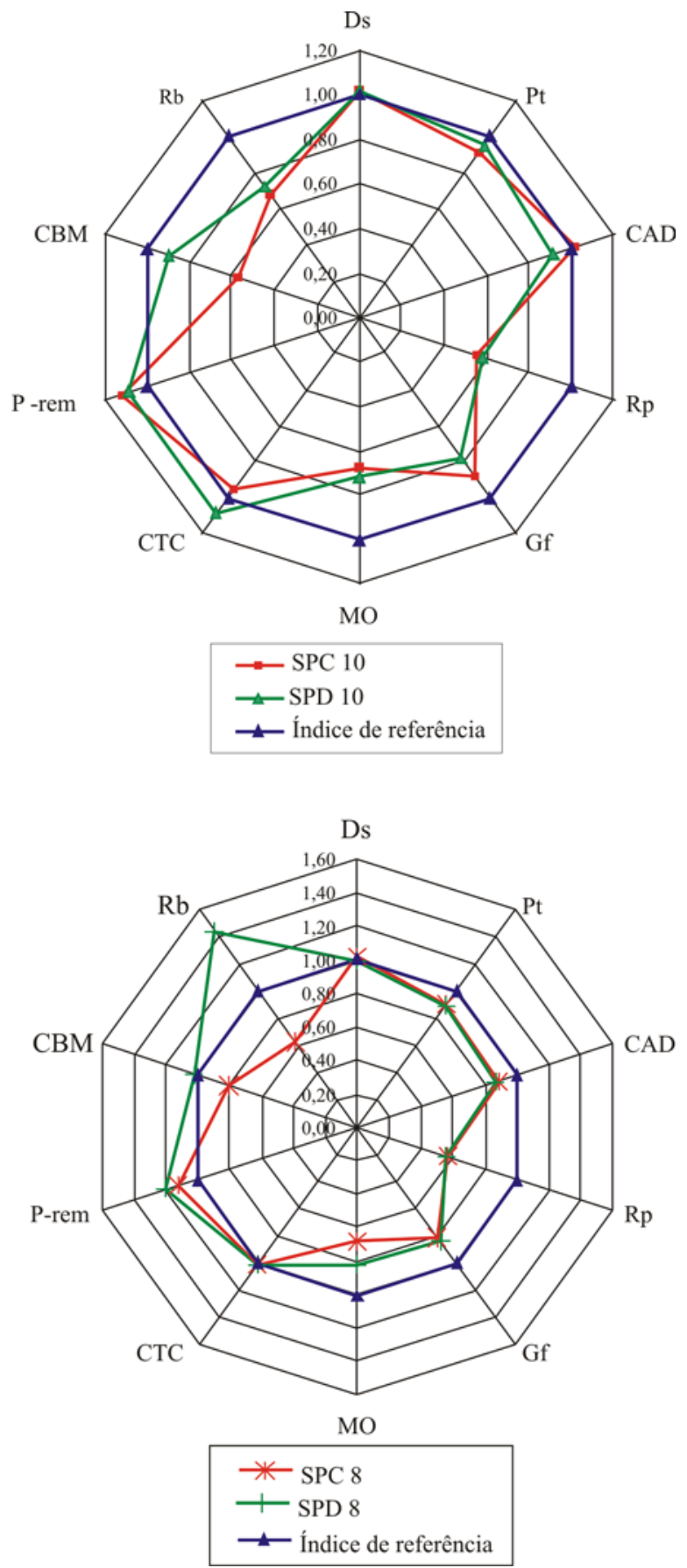

Figura 1. Diagrama comparativo de qualidade do solo sob sistemas de preparo convencional (SPC) e de plantio direto (SPD), em parcelas experimentais cultivadas por períodos de dez e oito anos, considerando-se a camada de solo de $0-10 \mathrm{~cm}$. Ds: densidade do solo; Pt: porosidade total do solo; CAD: capacidade de água disponível; Rp: resistência mecânica à penetração; Gf: grau de floculação; MO: matéria orgânica; CTC: capacidade de troca catiônica; P-rem: fósforo remanescente; CBM: carbono da biomassa microbiana; Rb: respiração basal. 
vegetação natural é rompido, e que um novo equilíbrio será estabelecido e, por isso, não parece pertinente a comparação da qualidade do solo cultivado com a do solo sob vegetação nativa.

\section{Conclusões}

1. Não há diferença entre a qualidade dos solos cultivados sob os sistemas de preparo convencional e plantio direto.

2. Os atributos biológicos são mais sensíveis para detectar a mudança de qualidade do solo sob cultivo.

3. A adoção de modelos gráficos radiais constitui-se instrumento útil na visualização comparativa da qualidade de solos.

4. O uso do solo sob vegetação de cerrado para a produção agrícola, independentemente do sistema de cultivo, resulta em modificações nos atributos de qualidade do solo.

\section{Referências}

ALVAREZ VENEGAS, V.H.; NOVAIS, R.F.; BARROS, N.F.; CANTARUTTI, R.B.; LOPES, A.S. Interpretação dos resultados das análises de solos. In: RIBEIRO, A.C.; GUIMARÃES, P.T.; ALVAREZ VENEGAS, V.H. (Ed.). Recomendações para o uso de corretivos e fertilizantes em Minas Gerais. Viçosa: UFV, 1999. p.25-32.

ARAÚJO, R. Avaliação da qualidade do solo em áreas sob diferentes usos. 2004. 82p. Dissertação (Mestrado) - Universidade de Brasília, Brasília.

BALOTA, E.L.; COLOZZI-FILHO, A.; ANDRADE, D.S.; HUNGRIA, M. Biomassa microbiana e sua atividade em solos sob diferentes sistemas de preparo e sucessão de culturas. Revista Brasileira de Ciência do Solo, v.22, p.641-649, 1998.

BEUTLER, A.N.; SILVA, M.L.N.; CURI, N.; FERREIRA, M.M.; CRUZ, J.C.; PEREIRA FILHO, I.A. Resistência à penetração e permeabilidade de Latossolo Vermelho distrófico típico sob sistemas de manejo na Região dos Cerrados. Revista Brasileira de Ciência do Solo, v.25, p.167-177, 2001.

D’ANDRÉA, A.F.; SILVA, M.L.N.; CURI, N.; SIQUEIRA, J.O.; CARNEIRO, M.A.C. Atributos biológicos indicadores da qualidade do solo em sistemas de manejo na Região do Cerrado do Sul do Estado de Goiás. Revista Brasileira de Ciência do Solo, v.26, p.913-923, 2002.
DUFRANC, G.; DECHEN, S.C.F.; FREITAS, S.S.; CAMARGO, O.A. Atributos físicos, químicos e biológicos relacionados com a estabilidade de agregados de dois latossolos em plantio direto no Estado de São Paulo. Revista Brasileira de Ciência do Solo, v.28, p.505-517, 2004.

EMBRAPA. Serviço Nacional de Levantamento e Conservação de Solos (Rio de Janeiro, RJ). Manual de métodos de análise de solos. 2.ed. Rio de janeiro, 1997. 212p.

FREITAS JÚNIOR, E.; SILVA, E.M. Uso da centrífuga para a determinação da curva de retenção de água no solo, em uma única operação. Pesquisa Agropecuária Brasileira, v.19, p.1423-1428, 1984.

GOEDERT, W.J.; SCHERMACK, M.J.; FREITAS, F.C. Estado de compactação do solo em áreas cultivadas no sistema de plantio direto. Pesquisa Agropecuária Brasileira, v.37, p.223-227, 2002.

JENKINSON, D.S.; POWLSON, D.S. The effects of biocidal treatments on metabolism in soil. V. A method for measuring soil biomass. Soil Biology and Biochemistry, v.8, p.209-213, 1976.

MARIA, I.C. de; CASTRO, O.M.; DIAS, H.S. Atributos físicos do solo e crescimento radicular de soja em Latossolo Roxo sob diferentes métodos de preparo do solo. Revista Brasileira de Ciência do Solo, v.23, p.703-709, 1999.

MENDES, I.C.; SOUZA, L.V.; RESCK, D.V.S.; GOMES, A.C. Propriedades biológicas em agregados de um Latossolo VermelhoEscuro sob plantio convencional e direto no Cerrado. Revista Brasileira de Ciência do Solo, v.27, p.435-443, 2003.

OLIVEIRA, G.C.; DIAS JUNIOR, M.S.; RESCK, D.V.S.; CURI, N. Caracterização química e físico-hídrica de um Latossolo Vermelho após vinte anos de manejo e cultivo do solo. Revista Brasileira de Ciência do Solo, v.28, p.327-336, 2004.

SECCO, D.; REINERT, D.J.; REICHERT, J.M.; ROS, C.O. da. Produtividade de soja e propriedades físicas de um Latossolo submetido a sistemas de manejo e compactação. Revista Brasileira de Ciência do Solo, v.28, p.797-804, 2004.

SEGUY, L.; KLUTHCOUSKI, J.; SILVA, J.G.; BLUMENSCHEIN, F.N.; DALL'ACQUA, F.M. Técnicas de preparo do solo: efeitos na fertilidade e na conservação do solo, nas ervas daninhas e na conservação de água. Goiânia: Embrapa-CNPAF, 1984. 26p.

SILVA, M.L.N.; CURI, N.; BLANCANEAUX, P. Sistemas de manejo e qualidade estrutural de Latossolo Roxo. Pesquisa Agropecuária Brasileira, v.35, p.2485-2492, 2000.

SOUSA, D.M.G. de; LOBATO, E. (Ed.). Cerrado: correção do solo e adubação. Planaltina: Embrapa Cerrados, 2004. 416p.

STONE, L.F.; SILVEIRA, P.M. Efeitos do sistema de preparo e da rotação de culturas na porosidade e densidade do solo. Revista Brasileira de Ciência do Solo, v.25, p.395-401, 2001. 